\section{An X-Ray Method for Studying Rapid Phase Changes in Steels at High Temperatures}

THE effects of temperature on transformations in steels have in the past usually been studied at room temperature after quenching. Thus, in a typical experiment, a specimen in the form of a rod was heated in such a manner as to establish a temperature gradient, so that the range of temperature in the specimen covered the region of interest. The rod was then quenched and zones (corresponding to particular temperatures at the time of quenching) examined by X-rays at room temperature. In this way information was obtained on the dependence of transformations on temperature. This method has two objectionable features. Primarily, the quench itself promotes transformations (austenite - martensite being a well-known example), so that the quenched material studied at room temperature is not the same as that prior to quenching. Secondly, the 'resolution' of the transformation is limited by the number of observations made.

Later workers have resorted to $\mathrm{X}$-ray studies at high temperatures, and several cameras have been designed in order to study transformations as they occur. These instruments have almost invariably incorporated photographic films, and the major difficulty here is the time factor. In order to make a detailed study of a phase change, exposures of the order of half an hour or more are required at a particular temperature. This presupposes that the temperature of the specimen is kept sensibly constant for this period. In order to study a transformation over a considerable range of temperature, many such exposures are required, and the time involved is consequently long.

In general, the $\mathrm{X}$-ray diffraction patterns of the phases present during the course of a transformation are well known. It is therefore unnecessary to record them completely in order to follow the course of the transformation. Invariably the phases of interest have isolated and unique diffraction angles. This fact is utilized in the method which is now in course of development in these Laboratories, in order to study transformations in steel.

In this method the specimen in the form of a thin plate approximately $1 \mathrm{~cm}$. square is mounted in an evacuated cylindrical furnace of length/diameter ratio of about 3, with the plane surface of the specimen parallel to the furnace axis. $\mathbf{X}$-Rays diverging from the focus of the X-ray tube, or from a slit in front of the tube window, irradiate one side of the specimen, entering and leaving through mica windows opposite the open ends of the cylindrical furnace. The low-angle diffracted radiation is approximately focused by the plane specimen on to the slit of a Geiger-Müller counter, which is situated so as to receive a strong diffraction line from the lowtemperature phase.

The pulses from the Geiger-Müller tube are fed to a recording counting-rate meter, in which for each discharge of the Geiger-Müller tube a fixed charge is conveyed to a parallel resistance-capacity circuit. The voltage across this circuit is amplified and operates a pen recorder, which thus provides a continuous record of the proportion of the low-temperature phase present. By raising the temperature and by simultaneously recording temperature and counts per second, the progress of the phase change may be studied, changes taking place in a time of the order of 1 second being clearly revealed. The use of low-angle scattering

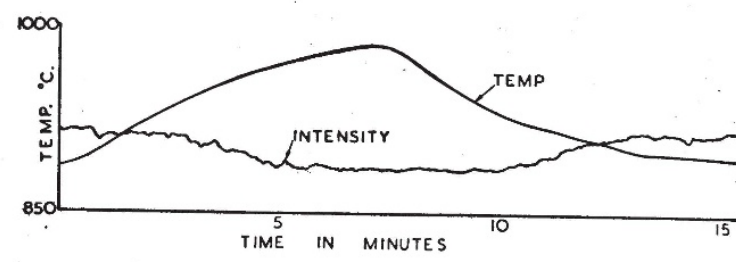

ensures that the angle is not appreciably affected by the thermal expansion of the specimen.

The method has been tested by observations on the (211) line from $\alpha$-iron at the $\alpha-\gamma$ transformation, a typical record being reproduced here. It is hoped at an early stage to record diffracted lines from lowand high-temperature phases simultaneously, and thereby increase the amount of information derived.

H. T. HEAL

Physics Laboratories,

J. SAVAgE

British Iron and Steel Research Association,

140 Battersea Park Road,

London, S.W.11. Jan. 11.

\section{An Automatic Recording Infra-Red Spectrometer}

WE have recently completed the construction of an infra-red spectrometer, which, by the automatic balancing of two beams of radiation from the same source, is designed to eliminate variations in the spectrum caused by atmospheric absorption, changes in source radiation intensity, and drifts in the electronic amplification. The infra-red absorption spectrum of any chemical compound can thus be recorded directly as percentage of radiation absorbed versus wave-length. If in solution, the solvent can be placed in the other beam, and the absorption due to the compound alone can thus be ascertained.

The general principles of this method have been given by Wright and Herscher ${ }^{1}$ and by Baird, O'Bryan, Ogden and $\mathrm{Lee}^{2}$. Radiation from the same source is caused to pass by two equivalent paths to the spectrometer entrance slit, and is interrupted at 10 c.p.s. in such a way that each beam alternately passes through the spectrometer entrance slit at that frequency. The spectrometer is of the Littrow type; the radiation thus passes through the prism twice, giving twice the dispersion. We are using a $60^{\circ}$ rock-salt prism of $10 \mathrm{~cm}$. base by $8 \mathrm{~cm}$. height supplied by Sir Howard Grubb, Parsons and Co., Ltd. The radiation passing through the exit slit is focused by the condenser mirror on to the receiving element of a Hilger-Schwarz fast vacuum thermopile, giving rise to a small signal voltage. Preliminary test experiments have been made using a lead sulphide cell as the detector, and these will now be extended by using the Hilger thermopile as detector.

The entrance and exit slits are a new type of our own design, and are opened and closed automatically by lever arms and cams mounted on the same gearbox which operates the Littrow mirror cam for wavelength scanning. The slits can be controlled accurately from zero up to $1.5 \mathrm{~mm}$.

The signal voltage from the detector is then amplified by a high-gain tuned A.C. amplifier, and rectified by mechanical switching in synchronism with the radiation chopper. The resulting D.c. voltage is then passed to a D.C. power amplifier which oper- 